\title{
Estrategias de aprendizaje y rendimiento académico en la asignatura Análisis Matemático II
}

\author{
LETICIA ENRIQUETA CHÁVEZ ARIAS \\ Escuela Superior Politécnica de Chimborazo - Ecuador \\ Recibido el 18-02-2017; primera evaluación el 06-11-2017; \\ segunda evaluación el 21-06-2018; aceptado el 02-07-2018
}

\section{RESUMEN}

El objetivo del estudio fue determinar la relación entre las estrategias de aprendizaje de los estudiantes de Ingeniería Mecánica de la Escuela Superior Politécnica de Chimborazo (ESPOCH) y el rendimiento académico en la asignatura Análisis Matemático II, con una población de 82 estudiantes. La investigación fue no experimental, con enfoque cuantitativo-cualitativo, de tipo descriptivo correlacional. Se utilizó el cuestionario CEVEAPEU para identificar las estrategias de aprendizaje y las notas finales en la asignatura para medir el rendimiento académico. El 52\% de estudiantes son de bajo rendimiento, el 48\% son de mediano rendimiento; diecinueve estrategias son las más utilizadas, cuatro inciden significativamente en el rendimiento académico, la estrategia planificación no utilizan los estudiantes de bajo rendimiento. Se rechaza la hipótesis de investigación y se recomienda realizar un estudio experimental sobre la estrategia de planificación, como predictor del rendimiento académico.

Palabras clave: estudiantes, estrategias de aprendizaje, aprovechamiento escolar.

Ingeniera mecánica. Especialista en Computación Aplicada al Ejercicio Docente. Magíster en Gerencia de Proyectos Educativos y Sociales. Magíster en Matemática Básica, Escuela Superior Politécnica de Chimborazo. Docente titular de la Escuela de Ingeniería Mecánica de la Escuela Superior Politécnica de Chimborazo (ESPOCH). Correo: leticia.chavez@espoch.edu.ec. 


\section{Learning strategies and academic performance in the subject Mathematical Analysis II}

\section{Abstract}

The objective was to determine the relationship between learning strategies for students at ESPOCH's School of Mechanical Engineering and the academic performance in Mathematical Analysis II by analyzing a sample population of 82 students. This research was not experimental, it was quantitative-qualitative and of the descriptive-correlational type. The CEVEAPEU questionnaire was used to identify learning strategies and final grades in order to measure academic performance. $52 \%$ of students were underperformers and there were no high performers. Nineteen learning strategies are predominant. Four strategies significantly impinged on academic performance. Only the "planning» learning strategy was not used by low performing students. The research hypothesis is rejected and it is recommended to conduct a pilot study on the "planning" learning strategy as a predictor of academic performance.

Keywords: students, learning strategies, academic performance.

\section{Estratégias de aprendizagem e desempenho acadêmico em Análise Matemática II}

\section{Resumo}

O objetivo foi determinar a relaçáo entre estratégias de aprendizagem dos alunos de Engenharia Mecânica ESPOCH e o desempenho acadêmico no assunto Análise Matemática II, com uma população (amostra) de 82 alunos. O estudo foi não experimental, com uma abordagem quantitativa-qualitativa, de tipo descritiva correlational. O questionário CEVEAPEU foi utilizado para identificar estratégias de aprendizagem e notas finais no assunto para medir o desempenho acadêmico. Aproximadamente $52 \%$ dos estudantes estão com baixo rendimento, e não há estudantes com alto desempenho; dezenove estratégias são as mais utilizadas e quatro imiscuiu significativamente no desempenho acadêmico, as estratégias de planejamento não usaram os alunos de baixo desempenho. A hipótese da pesquisa é rejeitada e se recomenda que um estudo piloto experimental sobre a estratégia de planejamento seja realizado, como preditor do desempenho acadêmico.

Palavras-chave: estudantes, estratégias de aprendizagem, desempenho acadêmico. 


\section{INTRODUCCIÓN}

La Facultad de Mecánica (FM) constituye una parte importante del entorno educativo de la Escuela Superior Politécnica de Chimborazo (ESPOCH) de la ciudad de Riobamba, Ecuador. En la actualidad, esta facultad cuenta con 4 carreras de ingeniería, cada una de ellas relacionadas de una u otra forma con la mecánica, estas son: industrial, automotriz, mantenimiento y mecánica, siendo esta última el objeto de estudio de la presente investigación.

Estas carreras y en especial la Escuela de Ingeniería Mecánica se han distinguido por sus características especiales, que las ubican en una posición competitiva en el mercado nacional; sin embargo, la mencionada escuela también ha presentado índices de reprobación importantes en el área de Matemática. Por citar un ejemplo, en el curso de Análisis Matemático II durante el semestre marzo-agosto 2013, en el paralelo "A», hubo $51 \%$ de reprobados y en el paralelo «B» 75\%; en el semestre septiembre 2013-enero 2014, en el paralelo "A», hubo 59\% de reprobados y en el paralelo "B» 63\%, tal como lo reflejan las actas de calificaciones que reposan en la secretaría académica de la Escuela.

En todos los niveles y ámbitos educativos, el problema del bajo rendimiento en los cursos de Matemática es general, y la Escuela de Ingeniería Mecánica de la ESPOCH no es la excepción. Esta problemática encierra una serie de aspectos relacionados con factores familiares, sociales, escolares e individuales, siendo este último parte de la temática de la que se ocupa la presente investigación, específicamente, respecto a las estrategias de aprendizaje del estudiante.

En el ámbito universitario se supone que el proceso de enseñanza se da en igualdad de condiciones para los estudiantes de un determinado curso; sin embargo, los resultados que se esperan no siempre son los mejores. Es notorio que entre los estudiantes hay diferencias tanto en el aprendizaje como en el rendimiento académico, como sucede en la asignatura de Análisis Matemático II.

Surgen entonces las siguientes interrogantes: ¿qué influye en el aprendizaje y en el rendimiento académico de los estudiantes?, ¿qué tipos de estrategias de aprendizaje están utilizando los mejores estudiantes?

Se encuentran muchas respuestas si se consideran los factores que inciden en el aprendizaje, tales como, conocimientos previos, aptitudes intelectuales, motivación, estrategias de aprendizaje, etc.; es así que una de las líneas de investigación que han cobrado importancia con el tiempo ha sido el que aborda las estrategias de aprendizaje. 
Según las investigaciones de varios autores (Román, 2004; Camarero, Martín y Herrero, 2000; Cano y Justicia, 1993; Bernard, 1999), se ha llegado a conclusiones que indican que estudiantes con alto rendimiento utilizan más y mejores estrategias de aprendizaje que aquellos de bajo rendimiento.

El bajo rendimiento académico de los estudiantes en la asignatura de Análisis Matemático II es un problema recurrente, de preocupación tanto personal como institucional, por lo que, en el presente trabajo, se trata de buscar una respuesta a la siguiente interrogante: ¿Qué incidencia tienen las estrategias de aprendizaje que utilizan los estudiantes de Ingeniería Mecánica de la ESPOCH en el rendimiento académico en la asignatura Análisis Matemático II?

El principal aporte de esta investigación reside en el hecho de que se van a identificar qué estrategias de aprendizaje están utilizando los estudiantes de Ingeniería Mecánica en la asignatura Análisis Matemático II, así como si hay diferencia en la utilización de estas estrategias entre estudiantes según su nivel de rendimiento y, finalmente, si estas estrategias están incidiendo significativamente en el rendimiento académico.

Esta investigación es un aporte a los esfuerzos por perfeccionar el nivel educativo en la Escuela de Ingeniería Mecánica, pues se trata de mejorar el rendimiento académico no solamente en la asignatura de Análisis Matemático II, sino en las restantes asignaturas del área.

\section{REVISIÓN TEÓRICA}

Una de las metas de una institución educativa es lograr el mejor aprendizaje posible de sus educandos, por lo que siempre se trata de buscar el modelo educativo que más se ajuste a sus necesidades, reconociendo que cada estudiante utiliza sus propios estilos y estrategias de aprendizaje.

Las estrategias de aprendizaje han sido tema de muchas investigaciones, tanto desde la investigación como desde la intervención. Varios modelos se han ido construyendo de acuerdo a los avances de este tipo de investigaciones, lo que ha dado paso a la construcción de varios instrumentos que permitan medir las estrategias de aprendizaje.

Al hablar de evaluación en el sistema educativo universitario, implícitamente se está hablando de evaluación de valores, actitudes y contenidos, lo cual es expresado mediante una nota que viene a convertirse en el referente del rendimiento académico. Es entonces importante tratar de determinar en forma específica cuáles son los factores que están incidiendo en el rendimiento académico, y, en forma particular, cuáles son manipulables desde la institución educativa. 
Respecto de un trabajo de investigación realizado en España, Tejedor y García (2007) mencionaron que, en opinión de los docentes, la variable que más incide en el bajo rendimiento de los estudiantes es el bajo nivel de conocimientos previos, una variable que es complicada de manejar, puesto que está relacionada con el quién, qué y cómo se impartió el contenido de las asignaturas previas. Se entiende, entonces, que la dificultad de manejar esta variable ha posibilitado que no se hayan realizado mayores estudios de este tema.

Gustavo Cáceres Cardeña (2009), en el trabajo de investigación denominado «Estrategias de aprendizaje de matemáticas en estudiantes de tercer semestre de preparatoria», tenía como objetivo identificar las estrategias de aprendizaje utilizadas por los estudiantes del tercer semestre, en la preparatoria uno y dos de la Universidad Autónoma de Yucatán, en la asignatura de matemáticas, y establecer el grado de correlación con el rendimiento académico de los estudiantes. Al concluir la investigación, se llegó a determinar que no existía una relación significativa entre estas dos variables.

En el trabajo de investigación llevado a cabo por Jorge Antonio Hilt (2011) acerca de qué tan predictoras son las estrategias de aprendizaje y las subescalas en el rendimiento académico de los estudiantes de licenciatura de Nuevo León, México, utilizando el cuestionario CEVEAPEU, se llegó a la conclusión de que, para medir las estrategias de aprendizaje, el cuestionario utilizado fue adecuado y existe una correlación positiva entre estas variables, lo que mejoró el valor predictivo, considerando variables como área académica, carrera, género y grado de avance de la carrera.

\subsection{Estrategias de aprendizaje}

Cuando se habla del aprendizaje, se habla de un cambio de conducta (conductismo/condicionamiento), de un cambio cognitivo (cognitivismo) y, de un proceso-resultado.

En principio, entendemos como aprendizaje lo dicho por Gargallo (2012): «un proceso activo de construcción de conocimientos, de adquisición de habilidades y estrategias de apropiación de actitudes y valores, (saber, saber hacer, saber ser y estar)».

Muchos autores, al hablar del progreso del aprendizaje de los estudiantes, coinciden en que uno de los elementos que más contribuye a este proceso es el modo en que estudian, es decir, qué y cuáles recursos cognitivos (estilos, enfoques y estrategias de aprendizaje) están utilizando durante el proceso de aprendizaje. 
En cuanto a número y nombre, los investigadores no han podido ponerse de acuerdo en la clasificación de estos procesos, pues cada uno de ellos los ha manejado de acuerdo a su criterio, pero en general se habla de procesos atencionales, de codificación, elaboración y organización, de retención, de recuperación y de respuesta.

Según Cárdenas (2004), las estrategias de aprendizaje pueden caracterizarse, en sentido general, destacando que:

- Son acciones específicas, o sistemas de acciones, determinadas por el estudiante.

- Están dirigidas al logro de un objetivo o solución de un problema determinado.

- Apoyan al aprendizaje de forma directa e indirecta.

- Presuponen la planificación y control de la ejecución.

- Implican el uso selectivo de los propios recursos y capacidades, lo que se relaciona con cierto nivel de desarrollo de las potencialidades cognitivas de los sujetos.

- Involucran a toda la personalidad y no solo su esfera cognitiva.

- Son flexibles.

- Son a menudo conscientes.

- No siempre son observables.

- Pueden enseñarse y resulta esencial el papel del profesor en este proceso.

Existen varios instrumentos para la evaluación de las estrategias de aprendizaje de los estudiantes, entre los cuales tenemos: Escala LASSI, de Weinstein, Schulte y Palmer (1987); Cuestionario español ACRA, de Román y Gallego (1994); Cuestionario de Estrategias de Aprendizaje y Motivación CEAM II como una adaptación al contexto español del instrumento MSLQ de Pintrich, Smith, Garcia y Mackeachie (1991) de los autores Roces, Tourón y González (1995); CEVEAPEU, el cuestionario que sirve como instrumento para evaluar las estrategias de aprendizaje de los estudiantes en la universidad, de los autores Gargallo, Suárez y Pérez (2009).

El cuestionario CEVEAPEU, según sus autores, es un instrumento que tiene mayores ventajas, a diferencia de los otros instrumentos mencionados anteriormente. Así, por ejemplo, el cuestionario español ACRA es solo validado en población no universitaria (12-16 años); la escala LASSI, diseñada para población universitaria estadounidense tiene limitaciones, puesto que, estrategias como la búsqueda y selección de información no se tomaron en cuenta, por citar un ejemplo. Por último, en la escala CEAM II, olvidaron estrategias cognitivas, como las de búsqueda, recogida y selección de 
información, no facilita la debida atención a las estrategias metacognitivas, entre otras limitaciones. Sin embargo, hay que destacar que las escalas mencionadas han servido como referente para la construcción del cuestionario que se utilizó en esta investigación (CEVEAPEU).

Los autores de la escala utilizada en este trabajo definen a las estrategias de aprendizaje como el manejo del grupo de técnicas y actividades organizadas que hace el estudiante de manera consciente y meditada para, en un contexto social, lograr con eficacia un objetivo de aprendizaje. Esta escala se basa en la clasificación de las estrategias de aprendizaje, dada en la Tabla 1.

Según los autores, esta división contiene los tres elementos fundamentales relacionados con el aprendizaje: voluntad, capacidad y autonomía (querer, poder y decidir) que, evidentemente, están ligados al concepto de estrategias de aprendizaje propuesto.

El cuestionario CEVEAPEU contiene dos escalas: 1) Las estrategias afectivas, de apoyo y de control y 2) Las estrategias cognitivas (relacionadas con el procesamiento de la información).

Las estrategias afectivas, de apoyo y de control tienen que ver con la parte afectiva y motivacional; afectiva en cuanto tiene relación con el estado emocional, psicológico y hasta físico del estudiante, así como su habilidad para interactuar tanto social como académicamente con sus compañeros; y motivacional, debido a la estimulación que tiene el estudiante en el proceso de aprendizaje.

La escala de estrategias cognitivas muestra las que están más directamente relacionadas con las estrategias de búsqueda, recolección, selección y procesamiento de la información.

\subsection{Rendimiento académico}

Para De Natale (1990), el aprendizaje y rendimiento implican la transformación del conocimiento. Define al rendimiento académico como un conjunto de habilidades, prácticas, ideales, anhelos, intereses, expectativas y realizaciones que el estudiante aplica en el proceso de aprendizaje.

El rendimiento académico es un constructo complejo en el que intervienen factores de orden personal, académico y social del estudiante, lo que influye directamente no solo en la calificación que puede obtener, como un valor cuantitativo de las materias aprobadas o no aprobadas, sino también en el grado de satisfacción personal que siente el estudiante.

Para Del Río (2001) citado por Pola (2013), el rendimiento académico es concebido como aquel proceso técnico pedagógico que califica los logros del estudiante en función de los objetivos de aprendizaje planteados. 
Tabla 1. Ítems de las estrategias de aprendizaje del CEVEAPEU

\begin{tabular}{|c|c|c|}
\hline \multirow[t]{4}{*}{$\begin{array}{l}\text { Estrategias } \\
\text { afectivas, } \\
\text { de apoyo y } \\
\text { control } \\
\text { (53 ítems) }\end{array}$} & $\begin{array}{l}\text { Estrategias motivacionales } \\
\text { (20 ítems) }\end{array}$ & $\begin{array}{l}\text { Motivación intrínseca (3 ítems) } \\
\text { Motivación extrínseca (2 ítems) } \\
\text { Valor de la tarea ( } 4 \text { ítems) } \\
\text { Atribuciones internas ( } 3 \text { ítems) } \\
\text { Atribuciones externas ( } 2 \text { ítems) } \\
\text { Autoeficacia y expectativas ( } 4 \text { ítems) } \\
\text { Concepción de la inteligencia como } \\
\text { modificable ( } 2 \text { ítems) }\end{array}$ \\
\hline & $\begin{array}{l}\text { Componentes afectivos } \\
\text { (8 ítems) }\end{array}$ & $\begin{array}{l}\text { Estado físico y anímico (4 ítems) } \\
\text { Ansiedad ( } 4 \text { ítems) }\end{array}$ \\
\hline & $\begin{array}{l}\text { Estrategias metacognitivas } \\
\text { (15 ítems) }\end{array}$ & $\begin{array}{l}\text { Conocimiento ( } 2 \text { ítems) } \\
\text { Planificación ( } 4 \text { ítems) } \\
\text { Autoevaluación (3 ítems) } \\
\text { Control, autorregulación (6 ítems) }\end{array}$ \\
\hline & $\begin{array}{l}\text { Estrategias de control de } \\
\text { contexto, interacción } \\
\text { social y manejo de recursos } \\
\text { (10 ítems) }\end{array}$ & $\begin{array}{l}\text { Control del contexto ( } 4 \text { ítems) } \\
\text { Habilidades de interacción social y apren- } \\
\text { dizaje con compañeros ( } 6 \text { ítems) }\end{array}$ \\
\hline \multirow[b]{2}{*}{$\begin{array}{l}\text { Estrategias } \\
\text { cognitivas } \\
\text { (relacionadas } \\
\text { con el } \\
\text { procesamiento } \\
\text { de la } \\
\text { información) } \\
\text { (35 ítems) }\end{array}$} & $\begin{array}{l}\text { Estrategias de búsqueda, } \\
\text { recolección y selección de } \\
\text { información ( } 8 \text { ítems) }\end{array}$ & $\begin{array}{l}\text { Conocimiento de fuentes ( } 4 \text { ítems) } \\
\text { Selección de información ( } 4 \text { ítems) }\end{array}$ \\
\hline & $\begin{array}{l}\text { Estrategias de } \\
\text { procesamiento y uso de la } \\
\text { información ( } 27 \text { ítems) }\end{array}$ & $\begin{array}{l}\text { Adquisición de información (3 ítems) } \\
\text { Elaboración (4 ítems) } \\
\text { Organización (5 ítems) } \\
\text { Personalización y creatividad (5 ítems) } \\
\text { Almacenamiento, memorización (3 ítems) } \\
\text { Almacenamiento. Simple repetición (2 } \\
\text { ítems) } \\
\text { Uso y transferencia de la información } \\
\text { adquirida (3 ítems) } \\
\text { Manejo de recursos para usar la informa- } \\
\text { ción adquirida ( } 2 \text { ítems) }\end{array}$ \\
\hline
\end{tabular}

Fuente: Gargallo, Suárez Rodríguez. y Pérez Pérez (2009). Clasificación de las estrategias de aprendizaje para la construcción del CEVEAPEU. 
$\mathrm{Al}$ valorar el rendimiento académico, las notas obtenidas por el estudiante en una determinada asignatura se constituyen en el indicador cuantitativo preciso de si se han alcanzado o no los logros en la parte personal, académica y social.

Según los investigadores del tema, es difícil obtener conclusiones del rendimiento académico de los estudiantes, pues se debe tener en cuenta que, si se refiere solo a las notas, se está hablando de un rendimiento académico inmediato, mientras que, si se habla de logros personales y profesionales, se estará refiriendo al rendimiento académico mediato, siendo este último cuantificable únicamente a medida que pase el tiempo.

Al respecto, Hernández de Rincón (2005) define conceptual y operativamente el rendimiento académico. Por un lado, como definición conceptual, presenta al rendimiento académico de los alumnos como el indicador de la productividad de un sistema educativo que suministra la data fundamental que activa y desata cualquier proceso evaluativo destinado a alcanzar una educación de calidad. Por otro lado, como definición operativa, menciona que el rendimiento académico es la media aritmética de las calificaciones de todos los alumnos de un docente que incluye solamente alumnos reprobados y aprobados.

Latiesa (1992), amplía el concepto de rendimiento académico relacionando las notas obtenidas por el estudiante con el éxito, el fracaso y la deserción. Al hablar de una calificación que obtiene el estudiante en determinada asignatura, esta es concebida como una valoración del rendimiento académico, que no es más que la relación entre lo que se aprende y se logra dentro del proceso de aprendizaje. En ese sentido, esta calificación es la sumatoria de las notas obtenidas en las diferentes actividades académicas que ha desarrollado el estudiante en un determinado período académico.

Es indiscutible que uno de los factores más influyentes en la calidad de la educación superior es el rendimiento académico de sus estudiantes y, en especial, en la educación pública, por la inversión estatal que conlleva. Por ello, resulta de vital importancia determinar cuáles son los factores que limitan y favorecen el desempeño de los estudiantes.

\section{Metodología}

La presente investigación se hizo con estudiantes de tercer semestre de Ingeniería Mecánica de la Escuela Superior Politécnica de Chimborazo, con una población de 82 estudiantes de ambos sexos, con edades comprendidas entre 19 y 22 años, matriculados en la asignatura Análisis Matemático II durante el 
período marzo-agosto 2014. Por su diseño, la investigación es no experimental transversal. Tiene un enfoque cuantitativo-cualitativo y es de tipo descriptivo correlacional. Para el desarrollo de la misma, se plantearon las siguientes hipótesis:

Hi: Las estrategias de aprendizaje de los estudiantes de Ingeniería Mecánica de la ESPOCH inciden significativamente en el rendimiento académico en la asignatura Análisis Matemático II.

Ho: Las estrategias de aprendizaje de los estudiantes de Ingeniería Mecánica de la ESPOCH no inciden significativamente en el rendimiento académico en la asignatura Análisis Matemático II.

Asimismo, para identificar las estrategias de aprendizaje de los estudiantes, se utilizó el cuestionario CEVEAPEU (Gargallo et al. 2009). Este instrumento está diseñado con el formato de las escalas tipo Likert con cinco opciones de respuesta: (A) muy en desacuerdo, (B) en desacuerdo, (C) indeciso, (D) de acuerdo, (E) muy de acuerdo. En la Tabla 1 se indican el número de ítems (88) que miden las veinticinco estrategias del instrumento. El rendimiento de los estudiantes en la asignatura Análisis Matemático II se midió a través de las notas finales obtenidas en la asignatura Análisis Matemático II en el período marzo-agosto 2014.

Para recoger los datos, se aplicó el instrumento a los dos paralelos al inicio del semestre marzo-agosto del 2014, previo consentimiento de los alumnos participantes, proporcionando las instrucciones sobre el uso y aplicación del material. Su aplicación fue de 120 minutos.

La información académica (notas finales de los estudiantes en la asignatura Análisis II del semestre marzo-agosto 2014) fue recabada de las actas de calificaciones de la secretaría de la Escuela de Ingeniería Mecánica.

Se recogió la información al aplicar el cuestionario CEVEAPEU y las notas finales de los estudiantes en la asignatura Análisis II, la cual fue tabulada y guardada en una base de datos en una hoja de Microsoft Excel, la misma que fue sistematizada para obtener toda la información estadística requerida.

Para definir el nivel de rendimiento académico, se tomó como base lo que se estipula en el artículo 194 del Reglamento General de la Ley Orgánica de Educación Intercultural Ecuatoriana en lo referente a la escala cualitativa de calificaciones, y se lo adaptó al régimen de evaluación y aprobación de asignaturas que consta en el Reglamento de Régimen Académico de la ESPOCH en sus artículos 68 y 72. Esta adaptación se indica en la Tabla 2. 
Tabla 2. Escala de calificaciones

\begin{tabular}{lcc}
\hline Escala cualitativa & \multicolumn{2}{c}{ Escala cuantitativa } \\
\cline { 2 - 3 } & (puntos) & $(\%)$ \\
\hline Domina los aprendizajes requeridos & $36-40$ & $90-100$ \\
Alcanza los aprendizajes requeridos & $28-35$ & $70-89$ \\
Está próximo a alcanzar los aprendizajes requeridos & $16-27$ & $40-69$ \\
No alcanza los aprendizajes requeridos & menor a 16 & Menor al 40 \\
\hline
\end{tabular}

Fuente: Elaboración propia sobre la base de la Ley Orgánica de Educación Intercultural Ecuatoriana y el Reglamento de Régimen Académico de la ESPOCH.

Para clasificar a los estudiantes según su nivel de rendimiento, se ha tomado en consideración lo descrito en la Tabla 2. Dicha clasificación se evidencia en la Tabla 3.

Tabla 3. Clasificación de los estudiantes

\begin{tabular}{lc}
\hline Clasificación & Calificaciones \\
\hline Alto rendimiento & $36-40(90-100 \%)$ \\
Mediano rendimiento & $28-35(70-89 \%)$ \\
Bajo rendimiento & Menor a 28 (70\%) \\
\hline
\end{tabular}

Fuente: Elaboración propia a partir de la información de la Tabla 2.

Para describir el rendimiento académico, se realizó un análisis de frecuencias de las notas finales de los estudiantes en la asignatura Análisis Matemático II, clasificándolos en alto, mediano y bajo rendimiento según la Tabla 3.

Para identificar cuáles son las estrategias de aprendizaje predominantes en los estudiantes, se procedió de la siguiente manera: si más del 50\% de estudiantes contestaba "de acuerdo" o «muy de acuerdo» en más del $50 \%$ de los ítems del cuestionario que correspondía a una de las 25 estrategias de aprendizaje, se asumió que el estudiante usa esta estrategia de aprendizaje. Por otro lado, si más del $50 \%$ de estudiantes contestaba «en desacuerdo» o «muy en desacuerdo» en más del $50 \%$ de los ítems del cuestionario que correspondía a una de las 25 estrategias de aprendizaje, se asumió que el estudiante no usa esta estrategia de aprendizaje.

Para identificar qué estrategias de aprendizaje estaban usando los estudiantes, se dividió a los mismos en dos grupos: mediano y bajo rendimiento, puesto que no se encontraron estudiantes de alto rendimiento. Para comprobar la hipótesis de investigación, se utilizó el estadístico Chi-cuadrado de independencia. 


\section{Resultados}

En el Gráfico 1, se puede observar que existen dos grupos distribuidos de forma casi equitativa en cuanto al rendimiento académico: los de bajo y mediano rendimiento, $52 \%$ y $48 \%$ respectivamente. No hay estudiantes de alto rendimiento.

\section{Gráfico 1. Rendimiento académico de los estudiantes}

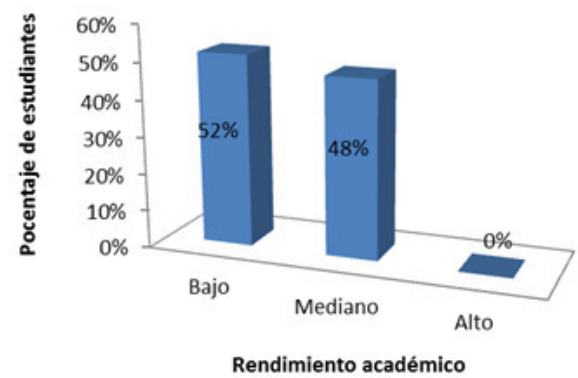

Fuente: Elaboración propia con datos de las actas de calificaciones de los estudiantes.

En el Gráfico 2, se puede observar que la gran mayoría de estudiantes $(86 \%)$ es de sexo masculino, y un pequeño grupo (14\%) es del sexo femenino, y la ubicación de estas estudiantes en bajo y mediano rendimiento conserva la misma tendencia que los estudiantes de sexo masculino.

Gráfico 2. Rendimiento académico de los estudiantes según el sexo

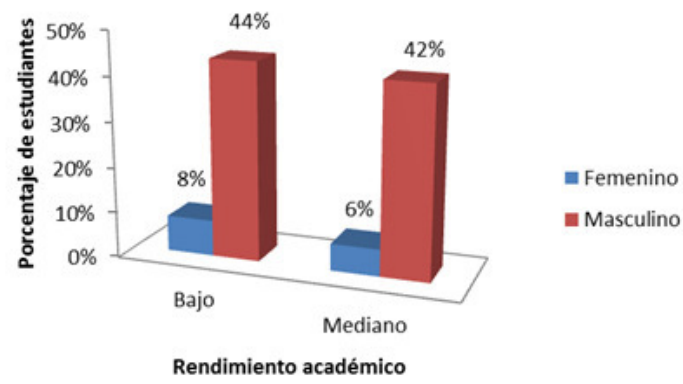

Fuente: Elaboración propia con datos de los encuestados.

En cuanto al rendimiento académico en función de la matrícula con la que estaban tomando la asignatura Análisis Matemático II, se puede observar, en el Gráfico 3, que de aquellos estudiantes que estaban tomando por primera vez la asignatura, el $35 \%$ es de bajo rendimiento y el 13\% de mediano rendimiento; 
de los que estaban tomando por segunda vez la asignatura, el 13\% es de bajo rendimiento y el $10 \%$ de mediano rendimiento, y, finalmente, de los que estaban tomando la asignatura por tercera vez, el 25\%, es de mediano rendimiento y el $4 \%$ es de bajo rendimiento.

Gráfico 3. Rendimiento académico de los estudiantes según la matrícula

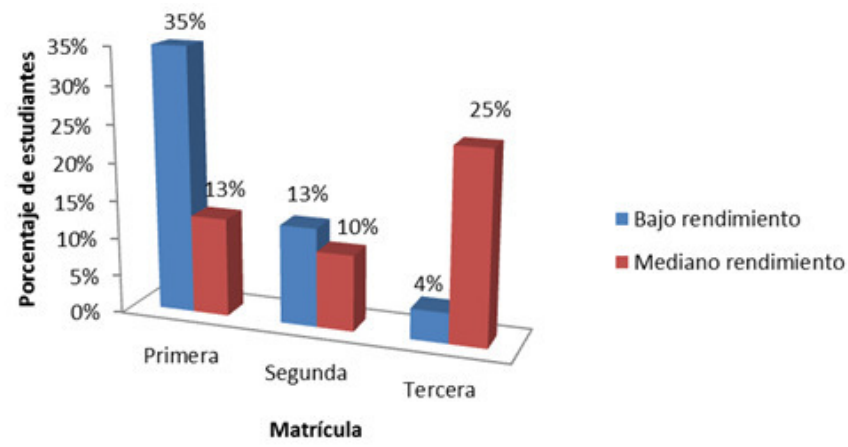

Fuente: Elaboración propia con datos de los encuestados.

Diecinueve estrategias son las predominantes en los estudiantes en cuanto a su utilización, apenas tres no son utilizadas (atribuciones externas, conocimiento de fuentes y búsqueda de información, y organización) y tres están divididas en cuanto a su utilización (concepción de la inteligencia como modificable, planificación y almacenamiento), puesto que, aproximadamente, la mitad de estudiantes las utilizan y la otra mitad no las utilizan.

Los estudiantes de mediano y bajo rendimiento no coincidían en la utilización de las siguientes estrategias: motivación extrínseca, ansiedad, planificación, organización y manejo de recursos para usar la información adquirida.

De acuerdo a la Tabla 5, las siguientes estrategias de aprendizaje inciden significativamente en el rendimiento académico de los estudiantes, para los restantes ítems no se rechaza la hipótesis: planificación, que mide el ítem 33, control y autorregulación, que mide el ítem 42 , habilidades de interacción social y aprendizaje con compañeros, que miden los ítems 48 y 52 y, manejo de recursos para usar la información adquirida, que mide el ítem 85.

\section{DISCUSIÓN, CONCLUSIONES Y RECOMENDACIONES}

Luego del análisis estadístico correspondiente, se determina que el 52\% de los 82 estudiantes quedan clasificados como de bajo rendimiento. Este resultado es coincidente con aquellos resultados obtenidos en investigaciones similares 
(Castillo y Bracamonte, 2011). Se observa, también, que no hay estudiantes de alto rendimiento. La mayoría de estudiantes que están tomando la asignatura con primera matrícula son de bajo rendimiento y la mayoría de estudiantes que están tomando la materia por tercera vez son de mediano rendimiento.

Tabla 4. Cuadro comparativo de las estrategias de aprendizaje predominantes en los estudiantes de mediano y bajo rendimiento

\begin{tabular}{|c|c|c|}
\hline Estrategia & $\begin{array}{c}\text { Utilizada por } \\
\text { los estudiantes } \\
\text { de mediano } \\
\text { rendimiento }\end{array}$ & $\begin{array}{c}\text { Utilizada por } \\
\text { los estudiantes } \\
\text { de bajo } \\
\text { rendimiento }\end{array}$ \\
\hline Motivación intrínseca & sí & sí \\
\hline Motivación extrínseca & sí/no & sí \\
\hline Valor de la tarea & sí & sí \\
\hline Atribuciones internas & sí & sí \\
\hline Atribuciones externas & no & no \\
\hline Autoeficacia y expectativas & sí & sí \\
\hline Concepción de la inteligencia como modificable & sí/no & sí/no \\
\hline Estado físico y anímico & sí & sí \\
\hline Ansiedad & sílno & sí \\
\hline Conocimiento de objetivos y criterios de evaluación & sí & sí \\
\hline Planificación & sí/no & no \\
\hline Autoevaluación & sí & sí \\
\hline Control, autorregulación & sí & sí \\
\hline Control del contexto & sí & sí \\
\hline $\begin{array}{l}\text { Habilidades de interacción social y aprendizaje con } \\
\text { compañeros }\end{array}$ & sí & sí \\
\hline Conocimiento de fuentes y búsqueda de información & sí/no & sí/no \\
\hline Selección de información & sí & sí \\
\hline Adquisición de información & sí & sí \\
\hline Elaboración & sí & sí \\
\hline Organización & no & sí \\
\hline Personalización y creatividad, pensamiento crítico & sí & sí \\
\hline $\begin{array}{l}\text { Almacenamiento. Memorización. Uso de recursos } \\
\text { mnemotécnicos }\end{array}$ & sí & sí \\
\hline Almacenamiento. Simple repetición & sí/no & sí/no \\
\hline Transferencia. Uso de la información & sí & sí \\
\hline Manejo de recursos para usar la información adquirida & sí & sí/no \\
\hline
\end{tabular}

Fuente: Elaboración propia con datos de los encuestados. 
Tabla 5. Resumen de las pruebas de independencia realizadas a cinco ítems del cuestionario CEVEAPEU

\begin{tabular}{ccccc}
\hline Ítem & Valor crítico & $\begin{array}{c}\text { Estadístico de } \\
\text { prueba }\end{array}$ & Valor P & $\begin{array}{c}\text { Decisión sobre la } \\
\text { hipótesis nula } \mathrm{H}_{0}\end{array}$ \\
\hline 33 & 9,48772904 & 11,3926158 & 0,02248849 & Se rechaza la hipótesis \\
42 & 5,99146455 & 6,07329793 & 0,04799545 & Se rechaza la hipótesis \\
48 & 9,48772904 & 11,167081 & 0,02474906 & Se rechaza la hipótesis \\
52 & 9,48772904 & 11,8715885 & 0,01833215 & Se rechaza la hipótesis \\
85 & 7,8147279 & 10,8853255 & 0,0123624 & Se rechaza la hipótesis \\
\hline
\end{tabular}

Fuente: Elaboración propia mediante la aplicación del estadístico Chi-cuadrado a los ítems del cuestionario CEVEAPEU.

En esta investigación, se trató de determinar si las estrategias de aprendizaje que están utilizando los estudiantes inciden significativamente en su rendimiento académico, mas, de acuerdo a los resultados obtenidos, se ve que no es así, al menos en lo relacionado a la cantidad de estrategias que están utilizando, aunque sí se ha encontrado que cuatro de las veinticinco estrategias están incidiendo en el rendimiento académico. Entonces, surge la siguiente interrogante; ¿cuáles otras variables están incidiendo en el rendimiento académico?

Como docente de la asignatura de Análisis Matemático II y coincidiendo con lo dicho por Tejedor y García (2007), se cree que, en este caso, la variable que está incidiendo en el bajo rendimiento es el escaso nivel de conocimientos previos del estudiante para cursar las asignaturas, pues la prueba diagnóstica de conocimientos previos así lo confirma.

De acuerdo a los resultados obtenidos en cuanto a la utilización o no de las estrategias de aprendizaje, los estudiantes de Análisis Matemático II están utilizando la mayoría de estrategias de aprendizaje y, al menos por la cantidad de estrategias que están utilizando (lo cual no garantiza que las conozcan bien y las estén utilizando adecuadamente), esta variable no está influyendo en su rendimiento. Esta realidad se diferencia de lo hallado en la investigación de Cáceres (2009), en la que todos los estudiantes manifestaron un nivel de uso de las estrategias de aprendizaje, de medio a bajo, lo cual sí estaba incidiendo en su rendimiento académico.

Tanto en el estudio realizado por Gargallo et al. (2009) como en el realizado por Hilt (2011), se encontraron que solo cinco de las veinticinco estrategias fueron consideradas como predictores del rendimiento académico. 
Sobre la base las pruebas de independencia de los 88 ítems para determinar si las estrategias relacionadas con estos ítems son significativas en el rendimiento académico de los estudiantes, se concluye que solo cuatro de las veinticinco estrategias están incidiendo significativamente en el rendimiento académico y estas son: planificación, que mide el ítem 33; control y autorregulación, que mide el ítem 42; habilidades de interacción social y aprendizaje con compañeros, que miden los ítems 48 y 52, y, finalmente, manejo de recursos para usar la información adquirida que mide el ítem 85 . De estas, solo la estrategia de planificación es la que no están utilizando los estudiantes de bajo rendimiento.

Entonces, se puede observar que hay diferencias y coincidencias con los autores antes mencionados en lo relacionado a que las estrategias de aprendizaje inciden en el rendimiento académico. Se entiende, por supuesto, que las investigaciones se realizaron en condiciones diferentes y, por eso, se dan estos resultados; sin embargo, se debe destacar que el instrumento utilizado en los tres casos es el cuestionario CEVEAPEU.

Por último, se recomienda efectuar un estudio experimental en la misma asignatura, Análisis Matemático II, u otras materias del área de matemática para determinar si efectivamente las estrategias de aprendizaje encontradas en esta investigación, especialmente la estrategia de planificación, están incidiendo significativamente en el rendimiento académico de los estudiantes.

\section{REFERENCIAS BIBLIOGRÁFICAS}

Bernard, J.A. (1999). Estrategias de aprendizaje. Madrid: Bruño.

Cáceres, G. (2009). Estrategias de aprendizaje de matemáticas en estudiantes de tercer semestre de preparatoria. Tesis de maestría no publicada, Universidad Autónoma de Yucatán, Yucatán, México.

Camarero, F., Martin, F. y Herrero, J. (2000). Estilos y estrategias de aprendizaje en estudiantes universitarios. Psicothema, 12(4), 615-622.

Cano, F. y Justicia, F. (1993). Factores académicos, estrategias y estilos de aprendizaje. Revista de Psicología General y Aplicada, 46(1), 89-99.

Cárdenas, N. (2004). ¿Cómo aprendo? Material utilizado en el postgrado «Enseñar a aprender» de la Maestría en Ciencias de la Educación, CEDE, UMCC, Matanzas.

Castillo, M. y Bracamonte, E. (2011). Estudio de la relación entre el estilo de aprendizaje de estudiantes de ingeniería y su rendimiento académico en matemática. XIII Conferencia Interamericana de Educación Matemática, Recife, Brasil. 
De Natale, M. L. (1990). Rendimiento escolar. Diccionario de Ciencias de la educación. Madrid: Paulinas.

Gargallo, B. (2012). Un aprendiz estratégico para una nueva sociedad. Teoría de la Educación. Educación y Cultura en la Sociedad de la Información, 13(2), 246-272.

Gargallo, B., Suárez, J. y Pérez, C. (2009). El cuestionario CEVEAPEU. Un instrumento para la evaluación de las estrategias de aprendizaje de los estudiantes universitarios. Relieve, 15(2), 1-31. Disponible en http://www. uv.es/RELIEVE/v15n2/RELIEVEv15n2_5.htm.

Gargallo, B., Suárez, J. y Pérez, C. (2009). Clasificación de las estrategias de aprendizaje para la construcción del CEVEAPEU.

Hernández de Rincón, A. (2005). El rendimiento académico de las matemáticas en alumnos universitarios. Encuentro Educacional, 12(1), 9-30.

Hilt, J. (2011). Estrategias de aprendizaje como predictoras del rendimiento académico en estudiantes de licenciatura del estado de Nuevo León, México. Tesis de maestría no publicada. Universidad de Montemorelos. Nuevo León, México.

Latiesa, M. (1992). La deserción universitaria. Madrid: Centro de Investigaciones Sociológicas.

Pintrich, P.R., Smith, D.A.F., Garcia, T. y Mackeachie, W.J. (1991). A Manual for Use of the Motivated Strategies for Learning Questionnaire (MSLQ). Technical Report No. 91-B-004. Ann Arbor: Universidad de Michigan.

Pola, A. (2013). Relación entre el rendimiento académico y los hábitos de estudio en alumnos universitarios (tesis de grado). Universidad Abierta Interamericana, Buenos Aires, Argentina.

Roces, C., Tourón, J. y González, M. C. (1995). Validación preliminar del CEAM II (Cuestionario de estrategias de aprendizaje y motivación II). Psicológica, 16(3), 347-366.

Román, J. M. y Gallego, S. (1994). ACRA. Escalas de estrategias de aprendizaje. Madrid: TEA.

Román, S., J.M. (2004). Procedimiento de aprendizaje autorregulado para universitarios: La estrategia de lectura sognificativa de textos. Revista Electrónica de Investigación Psicoeducativa, 2(1), 113-132.

Tejedor, F. y García, A. (2007). Causas del bajo rendimiento del estudiante universitario (en opinión de los profesores y alumnos). Propuestas de mejora en el marco del EEES. Revisa de Educación, 342, 443-473.

Weinstein, C., Schulte, A.C. y Palmer, D.R. (1987). Learning and Study Strategies Inventory (LASSI). Clearwater, FL: H \& H Publishing. 